\title{
Wave Interactions for the Suliciu Relaxation System*
}

\section{Bingzhuang Liu, Yujin Liü}

School of Mathematics and Statistics, Shandong University of Technology, Zibo, China

Email: *yjliu98@126.com

How to cite this paper: Liu, B.Z. and Liu, Y.J. (2021) Wave Interactions for the Suliciu Relaxation System. Journal of Applied Mathematics and Physics, 9, 1483-1496. https://doi.org/10.4236/jamp.2021.97100

Received: June 3, 2021

Accepted: July 10, 2021

Published: July 13, 2021

Copyright $\odot 2021$ by author(s) and Scientific Research Publishing Inc. This work is licensed under the Creative Commons Attribution International License (CC BY 4.0).

http://creativecommons.org/licenses/by/4.0/

\begin{abstract}
In this paper, we investigate the elementary wave interactions for the Suliciu relaxation system and construct uniquely the solution by the characteristic analysis method in the phase plane. We find that the elementary wave interactions have a much simpler structure for the Temple class than the general systems of conservation laws. It is observed that the Riemann solutions of the Suliciu relaxation system are stable under the small perturbation on the Riemann initial data.
\end{abstract}

\section{Keywords}

Hyperbolic Conservation Laws, Wave Interaction, Riemann Problem, Delta Shock, Suliciu Relaxation System

\section{Introduction}

In the present paper, we study the simplified viscoelastic shallow fluid model which is called the Suliciu relaxation system [1] [2] [3] [4]

$$
\left\{\begin{array}{l}
\rho_{t}+(\rho u)_{x}=0, \\
(\rho u)_{t}+\left(\rho u^{2}+s^{2} v\right)_{x}=0, \\
(\rho v)_{t}+(\rho u v+u)_{x}=0,
\end{array}\right.
$$

where $\rho \geq 0$ is the layer depth of fluid, $u$ is the horizontal velocity, $s>0$ is related to the stress tensor and is a conserved quantity, $v=\frac{\pi}{s^{2}}$ is the new variable which is concerned with the pressure and $\pi$ is the relaxed pressure.

In [2], the authors proposed the new reduced model for gravity-driven ${ }^{\star}$ Supported by the Foundation for Young Scholars of Shandong University of Technology (No. 115024).

${ }^{\#}$ Corresponding author. 
free-surface flows of shallow viscoelastic fluids, and established the mathematical properties for this model. In [3], the authors investigated the semilinear behavior of the totally linearly degenerate hyperbolic systems with relaxation, using pointwise estimates of the gradient, they obtained the semilinear behavior of the Suliciu model. In [4], the authors studied the qualitative behaviour of phase transition phenomena.

The above system can be considered as a relaxation for the following isentropic Chaplygin gas dynamics system [5] [6]

$$
\left\{\begin{array}{l}
\rho_{t}+(\rho u)_{x}=0, \\
(\rho u)_{t}+\left(\rho u^{2}+P\right)_{x}=0,
\end{array}\right.
$$

where $\rho, u$ are respectively the density and the velocity of the gas, the pressure $P$ is given by the state equation $P(\rho)=-\frac{s^{2}}{\rho}$ with $s>0$ a constant. It is found that (1) is of Temple class [7] and it is also of rich type but it is not diagonal.

The Riemann problem for the Suliciu relaxation system has been studied, for instance in [8] [9]. In [8], the authors are concerned with the numerical capture of stiff viscous shock solutions of the Navier-Stokes equations for complex compressible materials.

In [9], the authors obtained the delta shock wave solution under the generalized Rankine-Hugoniot relation and the entropy condition, they also constructed the explicit solution for the Cauchy problem with the initial data in $L^{\infty}$.

In the present paper, we investigate the elementary wave interactions for (1) with the following three piecewise constants

$$
(\rho, u, v)(x, 0)= \begin{cases}\left(\rho_{l}, u_{l}, v_{l}\right), & -\infty<x<-\varepsilon, \\ \left(\rho_{m}, u_{m}, v_{m}\right), & -\varepsilon<x<\varepsilon, \\ \left(\rho_{r}, u_{r}, v_{r}\right), & \varepsilon<x<+\infty,\end{cases}
$$

where the perturbation parameter $\varepsilon$ is sufficiently small. (3) can be regarded as a local perturbation on the initial values

$$
(\rho, u, v)(x, 0)=\left(\rho_{ \pm}, u_{ \pm}, v_{ \pm}\right), \quad \pm x>0 .
$$

By the characteristic analysis method, we analyze the positional relations of the elementary wave curves in the phase plane $(u, v)$ case by case. It is observed that for some cases even if there is no the delta shock wave in the corresponding Riemann solution, the delta shock wave occurs after the wave interaction. When there is the delta shock wave in the corresponding Riemann solution, the delta shock wave may disappear after the wave interaction. Since the system is of Temple class, the structure of the solution is simpler than the conventional gas dynamics. We find that the Riemann solutions of the initial value problem (1) and (4) are stable under the above small perturbation on the Riemann data.

This paper is arranged as follows. In Section 2, we give curtly the Riemann problem for the model (1) and (4) for the convenience of the readers. In Section 3 , we investigate the elementary wave interactions by the characteristic analysis method. In Section 4, we summarize our main conclusion. 


\section{Preliminaries}

We give briefly the Riemann problem for (1) with the initial values (4) (see [9]).

The characteristic roots of (1) are $\mu_{1}=u-\frac{s}{\rho}, \mu_{2}=u, \mu_{3}=u+\frac{s}{\rho}$, which shows that (1) is strictly hyperbolic. The corresponding right characteristic vector of $\mu_{1}, \mu_{2}$ and $\mu_{3}$ is respectively given by

$$
\vec{v}_{1}=\left(\rho^{2},-s, 1\right)^{\mathrm{T}}, \quad \vec{v}_{2}=(1,0,0)^{\mathrm{T}}, \quad \vec{v}_{3}=\left(\rho^{2}, s, 1\right)^{\mathrm{T}},
$$

we get

$$
\nabla \mu_{1} \cdot \vec{v}_{1}=0, \quad \nabla \mu_{2} \cdot \vec{v}_{2}=0, \quad \nabla \mu_{2} \cdot \vec{v}_{3}=0,
$$

which indicates that each characteristic field is linearly degenerate.

We construct the self-similar solution $(\rho, u, v)(x, t)=(\rho, u, v)(\zeta), \quad \zeta=\frac{x}{t}$. The Riemann problem (1) and (4) become the following boundary value problem of the ordinary differential equations

$$
\left\{\begin{array}{l}
-\zeta \rho_{\zeta}+(\rho u)_{\zeta}=0 \\
-\zeta(\rho u)_{\zeta}+\left(\rho u^{2}+s^{2} v\right)_{\zeta}=0 \\
-\zeta(\rho v)_{\zeta}+(\rho u v+u)_{\zeta}=0
\end{array}\right.
$$

and $(\rho, u, v)( \pm \infty)=\left(\rho_{ \pm}, u_{ \pm}, v_{ \pm}\right)$. For smooth solutions, let $V=(\rho, u, v)^{\mathrm{T}}$, (7) becomes

$$
A(V) V_{\zeta}=0
$$

where

$$
A(V)=\left(\begin{array}{ccc}
u-\zeta & \rho & 0 \\
0 & \rho(u-\zeta) & 0 \\
0 & 1 & \rho(u-\zeta)
\end{array}\right)
$$

Besides the constant state solution $(\rho, u, v)=$ constant, (8) has a rarefaction wave solution. For the given left state $\left(\rho_{-}, u_{-}, v_{-}\right)$, the rarefaction wave curve is given by

$$
\bar{R}\left(\rho_{-}, u_{-}, v_{-}\right):\left\{\begin{array}{l}
\zeta=\mu_{1}=u-\frac{s}{\rho}, \\
u=u_{-} s\left(v-v_{-}\right), \\
v<v_{-}, u>u_{-} .
\end{array}\right.
$$

For a bounded discontinuity at $\zeta=\tau$, it holds the Rankine-Hugoniot conditions

$$
\left\{\begin{array}{l}
-\tau[\rho]+[\rho u]=0 \\
-\tau[\rho u]+\left[\rho u^{2}+s^{2} v\right]=0 \\
-\tau[\rho v]+[\rho u v+u]=0
\end{array}\right.
$$

where $[\rho]=\rho_{+}-\rho_{-}, \rho_{-}=\rho(\tau-0), \rho_{+}=\rho(\tau+0)$, etc.

For the given left state $\left(\rho_{-}, u_{-}, v_{-}\right)$, the shock wave is given by 


$$
\bar{S}\left(\rho_{-}, u_{-}, v_{-}\right):\left\{\begin{array}{l}
\tau=u-\frac{s}{\rho}, \\
u=u_{-}-s\left(v-v_{-}\right), \\
v>v_{-}, u<u_{-} .
\end{array}\right.
$$

From (10) we know the contact discontinuity

$$
J: u=u_{-}, v=v_{-},[\rho] \neq 0 .
$$

All the above rarefaction waves $R$, shock waves $S$, and contact discontinuities $J$ are the elementary waves for (1). Notice the shock curves coincide with the rarefaction curves in the phase plane $(\rho, u, v)$ [7]. It is very important because it can simplify the process of the elementary wave interactions.

We give the backward wave curves in the $(u, v)$ plane (Figure 1), where the straight line $l_{1}$ is given by $v=\frac{1}{s}\left[u-\left(u_{-}+s v_{-}\right)\right]$, and the point $A\left(u_{-}+s v_{-}, 0\right)$. When $\left(u_{+}, v_{+}\right) \in \mathrm{I}$, the unique Riemann solution is $\overleftarrow{S}+J+\vec{R}$; when $\left(u_{+}, v_{+}\right) \in \mathrm{II}$, the unique Riemann solution is $\overleftarrow{S}+J+\vec{S}$; when $\left(u_{+}, v_{+}\right) \in \mathrm{III}$, the unique Riemann solution is $\overleftarrow{R}+J+\vec{S}$; when $\left(u_{+}, v_{+}\right) \in \mathrm{IV}$, the unique Riemann solution is $\overleftarrow{R}+J+\vec{R}$.

The forward wave curves in the $(u, v)$ plane can be given similarly (Figure $2)$, where $l_{2}$ is given by $v=-\frac{1}{s}\left[u-\left(u_{+}-s v_{+}\right)\right]$, and the point $B\left(u_{+}-s v_{+}, 0\right)$.

When $\left(u_{+}, v_{+}\right) \in \mathrm{V}$, i.e., $u_{+}+\frac{s}{\rho_{+}} \leq u_{-}-\frac{s}{\rho_{-}}$, we should construct the delta shock wave solution as follows.

Consider a piecewise smooth solution of (1) with the form

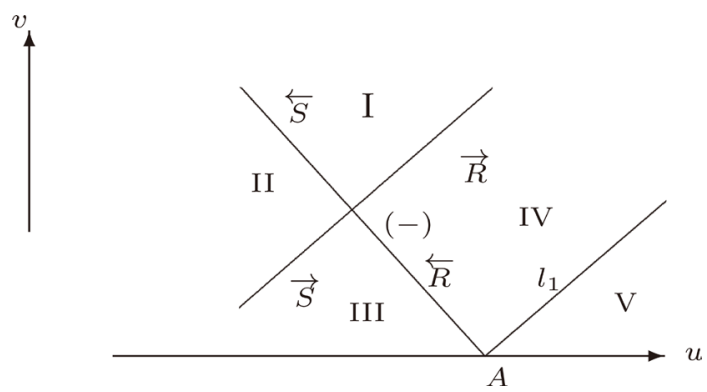

Figure 1. Backward wave curves in $(u, v)$.

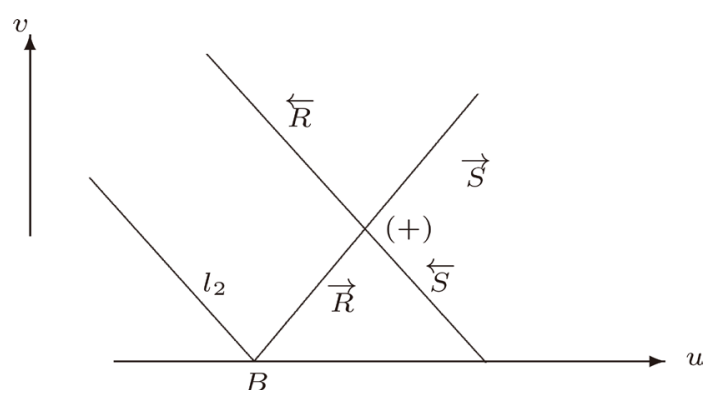

Figure 2. Forward wave curves in $(u, v)$. 


$$
(\rho, u, v)(x, t)= \begin{cases}\left(\rho_{-}, u_{-}, v_{-}\right), & x<x(t), \\ \left(\omega(t) \delta(x-x(t)), u_{\delta}(t), g(t)\right), & x=x(t), \\ \left(\rho_{+}, u_{+}, v_{+}\right), & x>x(t),\end{cases}
$$

where $\delta(x-x(t))$ is the $\delta$ measure on the support of $x=x(t), \omega(t)$ denotes the weight of the delta wave $x=x(t)$. Let $\frac{\mathrm{d} x}{\mathrm{~d} t}=u_{\delta}(t)$, since the concentration in $\rho$ needs to travel at the speed of discontinuity. The above delta shock wave is a measure solution to the Suliciu relaxation system if and only if the following generalized Rankine-Hugoniot condition holds

$$
\left\{\begin{array}{l}
\frac{\mathrm{d} x(t)}{\mathrm{d} t}=u_{\delta}(t), \\
\frac{\mathrm{d} \omega(t)}{\mathrm{d} t}=-[\rho] u_{\delta}(t)+[\rho u], \\
\frac{\mathrm{d}\left(\omega(t) u_{\delta}(t)\right)}{\mathrm{d} t}=-[\rho u] u_{\delta}(t)+\left[\rho u^{2}+s^{2} v\right], \\
\frac{\mathrm{d}(\omega(t) g(t))}{\mathrm{d} t}=-[\rho v] u_{\delta}(t)+[\rho u v+u],
\end{array}\right.
$$

where $[u]=u(x(t)+0)-u(x(t)-0)$ is the jump of $u$ across the discontinuity $x=x(t)$, etc.

The $\delta$-entropy condition is

$$
\mu_{3}\left(\rho_{+}, u_{+}, v_{+}\right) \leq u_{\delta}(t) \leq \mu_{1}\left(\rho_{-}, u_{-}, v_{-}\right),
$$

which is

$$
u_{+}+\frac{s}{\rho_{+}} \leq u_{\delta}(t) \leq u_{-}-\frac{s}{\rho_{-}} .
$$

When $[\rho]=0$, we have

$$
\begin{aligned}
& \qquad\left\{\begin{array}{l}
u_{\delta}=\frac{u_{+}+u_{-}}{2}+s^{2} \frac{v_{+}-v_{-}}{2 \rho_{-}\left(u_{+}-u_{-}\right)}, \\
x(t)=u_{\delta} t, \\
\omega(t)=\rho_{-}\left(u_{-}-u_{+}\right) t, \\
g(t)=\frac{\left(\rho_{+} u_{+} v_{+}+u_{+}-\rho_{-} u_{-} v_{-}-u_{-}\right)-u_{\delta}}{\rho_{+} u_{+}-\rho_{-} u_{-}} t,
\end{array}\right. \\
& \left\{\begin{array}{l}
\text { When }[\rho] \neq 0, \text { we have } \\
u_{\delta}=\frac{\left(\rho_{+} u_{+}-\rho_{-} u_{-}\right)-\sqrt{\left(\rho_{+} u_{+}-\rho_{-} u_{-}\right)^{2}-\left(\rho_{+}-\rho_{-}\right)\left(\rho_{+} u_{+}^{2}+s^{2} v_{+}-\rho_{-} u_{-}^{2}-s^{2} v_{-}\right)}}{\rho_{+}-\rho_{-}}, \\
\omega(t)=\sqrt{\left(\rho_{+} u_{+}-\rho_{-} u_{-}\right)^{2}-\left(\rho_{+}-\rho_{-}\right)\left(\rho_{+} u_{+}^{2}+s^{2} v_{+}-\rho_{-} u_{-}^{2}-s^{2} v_{-}\right)} \cdot t, \\
g(t)=\frac{-[\rho v][\rho u]+[\rho v] \sqrt{\left(\rho_{+} u_{+}-\rho_{-} u_{-}\right)^{2}-\left(\rho_{+}-\rho_{-}\right)\left(\rho_{+} u_{+}^{2}+s^{2} v_{+}-\rho_{-} u_{-}^{2}-s^{2} v_{-}\right)}+[\rho][\rho u v+u]}{\left(\rho_{+}-\rho_{-}\right) \sqrt{\left(\rho_{+} u_{+}-\rho_{-} u_{-}\right)^{2}-\left(\rho_{+}-\rho_{-}\right)\left(\rho_{+} u_{+}^{2}+s^{2} v_{+}-\rho_{-} u_{-}^{2}-s^{2} v_{-}\right)}},
\end{array}\right.
\end{aligned}
$$


Based on the above analysis, we have the following conclusion.

Theorem 2.1. The Riemann solution of the initial value problem (1) with the initial data (4) exists uniquely.

\section{Interactions of Elementary Waves}

Now we study the elementary wave interactions for (1) with the initial data (3). We construct the unique solution with the characteristic analysis method in the phase plane (see [10] [11]). (3) is regarded as the perturbation on the Riemann initial values (4). In order to cover all the cases containing no delta shock, we have three possibilities according to the different combinations from $(-\varepsilon, 0)$ and $(\varepsilon, 0)$ as follows. $\vec{W}$ and $\bar{W}, \vec{W}$ and $\vec{W}, \vec{W}$ and $J$, where $\vec{W}=\vec{R} \cup \vec{S}, \bar{W}=\bar{R} \cup \bar{S}$.

Case 1: The collision of $\vec{W}$ and $\bar{W}$.

We consider the collision wave interaction of a forward wave $\vec{W}$ emanating from $(-\varepsilon, 0)$ and a backward wave $\bar{W}$ emanating from $(\varepsilon, 0)$. There are five subcases as follows.

Subcase $1.1 \vec{R}$ and $\bar{R}$ and $v_{l}<v_{m}, v_{r}<v_{m}, v_{*}>0$ (Figure 3).

Subcase 1.2 $\vec{S}$ and $\bar{R}$ and $v_{l}>v_{m}>v_{r}$ (Figure 4).

Subcase $1.3 \vec{R}$ and $\bar{S}$ and $v_{r}>v_{m}>v_{l}$ (Figure 5).

Subcase $1.4 \vec{S}$ and $\bar{S}$ and $v_{l}>v_{m}, v_{r}>v_{m}$ (Figure 6).

Subcase $1.5 \vec{R}$ and $\bar{R}$ and $v_{l}>v_{m}, v_{r}>v_{m}, v_{*} \leq 0$ (Figure 8).

The discussions for Subcase 1.1, Subcase 1.2, Subcase 1.3 are similar with the discussions for Subcase 1.4, thus we just need to consider Subcase 1.4 and Subcase 1.5.

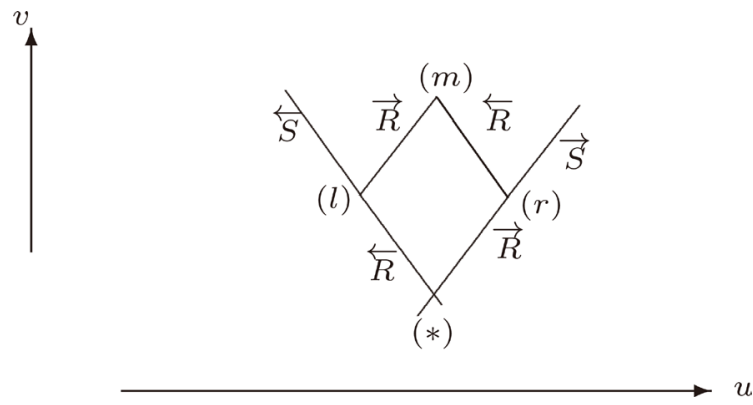

Figure 3. Collision of $\vec{R}$ and $\bar{R}, v_{*}>0$.

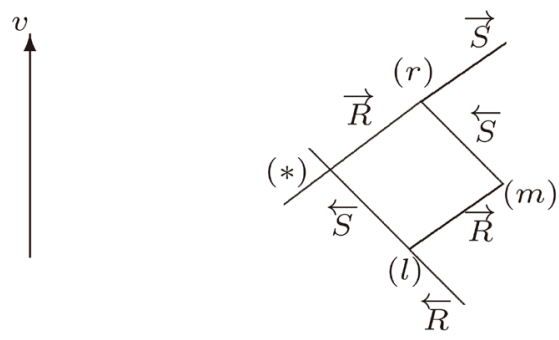

Figure 4. Collision of $\vec{R}$ and $\bar{S}$. 


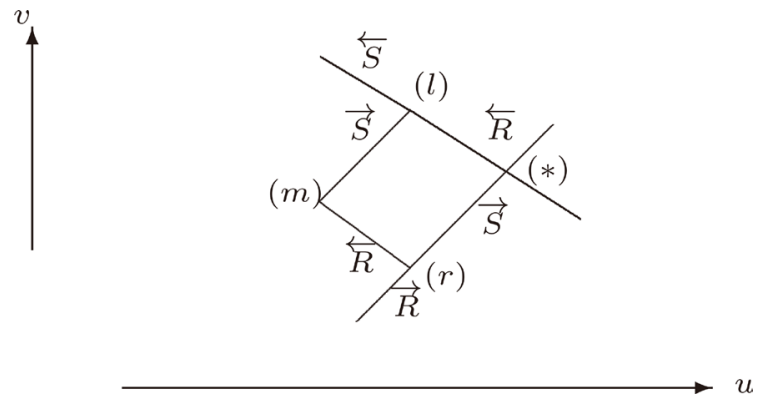

Figure 5. Collision of $\vec{S}$ and $\bar{R}$.

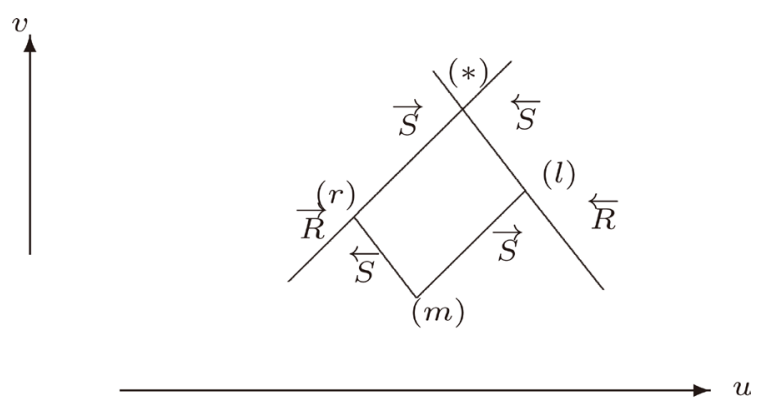

Figure 6. Collision of $\vec{S}$ and $\bar{S}$.

Subcase 1.4 $\vec{S}$ and $\bar{S}$. The forward shock wave $\vec{S}$ emanating from $(-\varepsilon, 0)$ and the backward shock wave $\bar{S}$ emanating from $(\varepsilon, 0)$ (Figure 6). Since the wave speed $\sigma_{1}$ of $\vec{S}$ is greater than the wave speed $\sigma_{2}$ of $\bar{S}$, they will interact with each other at the finite time $t=t_{0}$ and a new Riemann problem will be formed with $(I)$ the left state and $(r)$ the right state.

Draw the backward wave curve $\bar{W}(l)$ from $(I)$ and draw the forward wave curve $\vec{W}(r)$ from $(r)$, it follows that they intersect with each other at $\left.{ }^{*}\right)$. It is found that the unique solution of the new Riemann problem is given by (Figure 7)

$$
\vec{S}+\bar{S} \rightarrow \bar{S}+J+\vec{S} \text {. }
$$

Subcase $1.5 \vec{R}$ and $\bar{R}, v_{*} \leq 0$. The forward rarefaction wave $\vec{R}$ emanating from $(-\varepsilon, 0)$ and the backward rarefaction wave $\bar{r}$ emanating from $(\varepsilon, 0)$ (Figure 8 ). Since the wave speed $\sigma_{3}$ of $\vec{R}$ is greater than the wave speed $\sigma_{4}$ of $\bar{R}$, they will interact with each other at the finite time $t=t_{0}$ and a new Riemann problem will be formed with $(I)$ the left state and $(r)$ the right state.

Draw the backward wave curve $\bar{W}(l)$ from $(I)$ and draw the forward wave curve $\vec{W}(r)$ from $(r)$, it is found that the solution of the new Riemann problem is the delta shock wave solution (Figure 9)

$$
\vec{R}+\bar{R} \rightarrow \delta .
$$

When $\rho_{l}=\rho_{r}$, it follows that

$$
\left\{\begin{array}{l}
u_{\delta}=\frac{u_{l}+u_{r}}{2}+s^{2} \frac{v_{r}-v_{l}}{2 \rho_{l}\left(u_{r}-u_{l}\right)}, \\
\omega(t)=\rho_{l}\left(u_{l}-u_{r}\right)\left(t-t_{0}\right),
\end{array}\right.
$$




$$
\vec{S}+\overleftarrow{S} \rightarrow \overleftarrow{S}+J+\vec{S}
$$

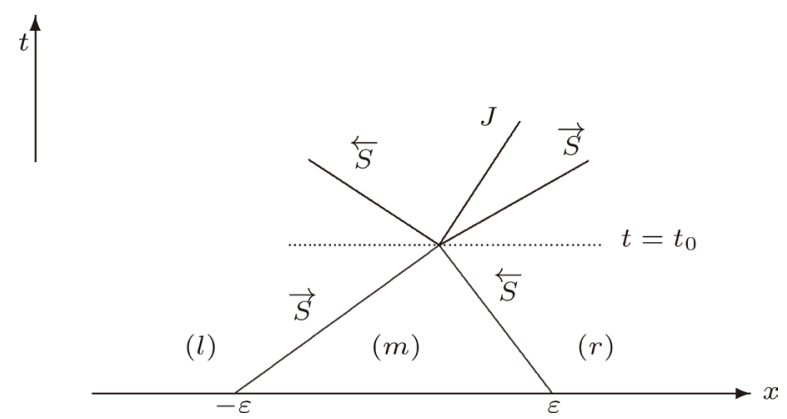

Figure 7. Interaction of $\vec{S}$ and $\bar{S}$.

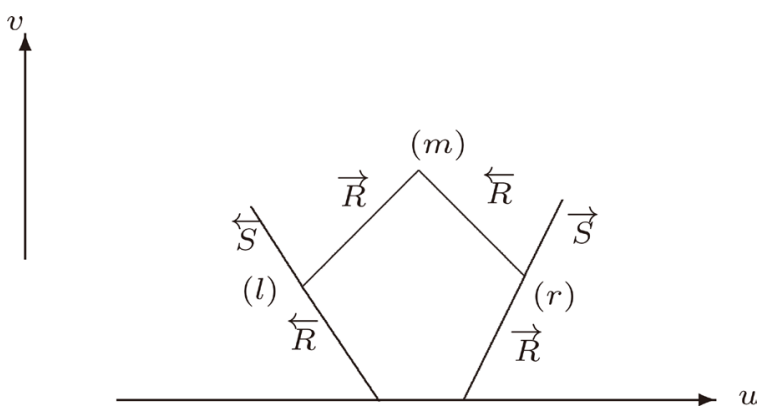

Figure 8. Collision of $\vec{R}$ and $\bar{R}, \quad v_{*} \leq 0$.

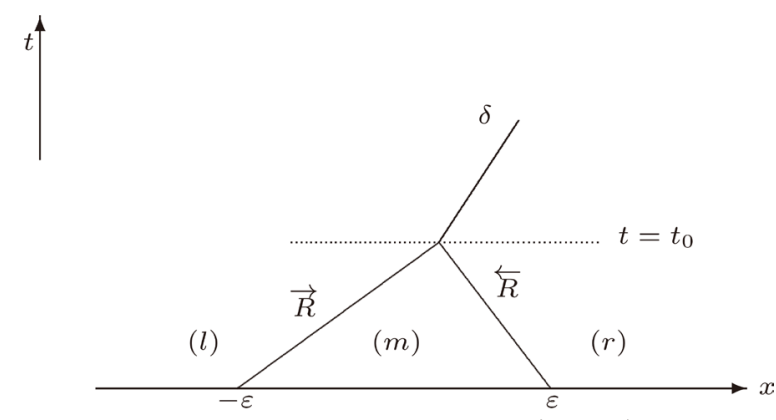

Figure 9. Interaction of $\vec{R}$ and $\bar{R}, v_{*} \leq 0$.

when $\rho_{l} \neq \rho_{r}$, it follows that

$$
\left\{\begin{array}{l}
u_{\delta}=\frac{\left(\rho_{r} u_{r}-\rho_{l} u_{l}\right)-\sqrt{\left(\rho_{r} u_{r}-\rho_{l} u_{l}\right)^{2}-\left(\rho_{r}-\rho_{l}\right)\left(\rho_{r} u_{r}^{2}+s^{2} v_{r}-\rho_{l} u_{l}^{2}-s^{2} v_{l}\right)}}{\rho_{r}-\rho_{l}}, \\
\omega(t)=\sqrt{\left(\rho_{r} u_{r}-\rho_{l} u_{l}\right)^{2}-\left(\rho_{r}-\rho_{l}\right)\left(\rho_{r} u_{r}^{2}+s^{2} v_{r}-\rho_{l} u_{l}^{2}-s^{2} v_{l}\right)} \cdot\left(t-t_{0}\right) .
\end{array}\right.
$$

Theorem 3.1. When the forward shock wave intersects with the backward shock wave, the backward shock wave will propagate in its original direction and the forward shock wave will also propagate in its original direction. When the forward rarefaction (or shock) wave intersects with the backward shock (or rarefaction) wave, the forward rarefaction (or shock) wave will propagate in its original direction and the backward shock (or rarefaction) wave will also propagate in its original direction, and the contact discontinuity will occur after the 
wave intersection. When the forward rarefaction wave intersects with the backward rarefaction wave, we see that there are two possibilities. One is that the backward rarefaction wave will propagate in its original direction and the forward rarefaction wave will also propagate in its original direction, the other one is that the solution of this new Riemann problem is the delta shock wave. The above conclusions tell us that the Riemann solution of (1) with the initial data (4) are stable for this case.

\section{Case 2: The overtaking of $\vec{W}$ and $\vec{W}$.}

Suppose the forward wave $\vec{W}$ which connects the left state and the middle state is given by

$$
\left\{\begin{array}{l}
u_{m}=u_{l}+s\left(v_{m}-v_{l}\right), \\
v_{m}+\frac{1}{\rho_{m}}=v_{l}+\frac{1}{\rho_{l}},
\end{array}\right.
$$

the forward wave $\vec{W}$ which connects the middle state and the right state is given by

$$
\left\{\begin{array}{l}
u_{r}=u_{m}+s\left(v_{r}-v_{m}\right), \\
v_{r}+\frac{1}{\rho_{r}}=v_{m}+\frac{1}{\rho_{m}} .
\end{array}\right.
$$

It is observed that the forward wave $\vec{W}$ and the forward wave $\vec{W}$ both pass through the middle state $(m)$, and the slope of them is equal, that is, the left state, the middle state and the right state are on the same wave curve. Then we have the solution of the new Riemann problem is given as $\vec{W}+\vec{W} \rightarrow \vec{W}$.

We give the detailed results for this case as follows.

Subcase 2.1 $\vec{S}+\vec{S}$ (Figure 10).

For this subcase, we obtain that the unique solution of the wave interaction is given by $\vec{S}+\vec{S} \rightarrow \vec{S}$.

Subcase $2.2 \vec{S}+\vec{R}$ (Figure 11).

For this subcase, we have the unique solution of the wave interaction $\vec{S}+\vec{R} \rightarrow \vec{S}$ or $\vec{R}$.

Subcase 2.3 $\vec{R}+\vec{S}$ (Figure 12).

For this subcase, we have the unique solution of the wave interaction $\vec{R}+\vec{S} \rightarrow \vec{R}$ or $\vec{S}$.

Subcase $2.4 \vec{R}+\vec{R}$ (Figure 13).

For this subcase, we obtain that the unique solution of the wave interaction is given by $\vec{R}+\vec{R} \rightarrow \vec{R}$.

Theorem 3.2. When the forward shock (or rarefaction) wave overtakes the forward shock (or rarefaction) wave, the solution of the wave interaction is still the forward shock (or rarefaction) wave which will propagate in its original direction. When the forward shock (or rarefaction) wave overtakes the forward rarefaction (or shock) wave, the solution of the wave interaction is the forward shock wave or the forward rarefaction wave. The above conclusions reveal that the Riemann solution of (1) with the initial data (4) are stable for this case. 


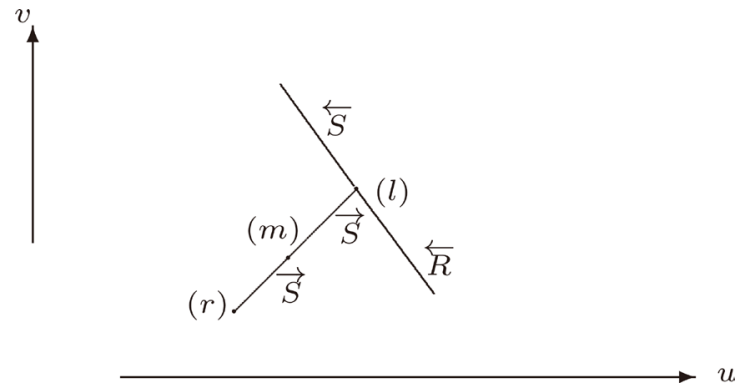

Figure 10. Overtaking of $\vec{S}$ and $\vec{S}$.
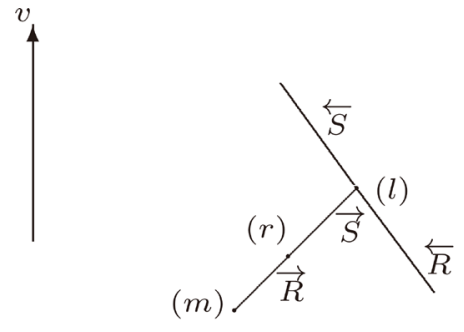

Figure 11. Overtaking of $\vec{S}$ and $\vec{R}$.

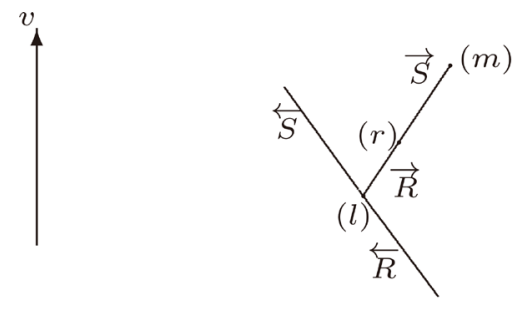

Figure 12. Overtaking of $\vec{R}$ and $\vec{S}$.

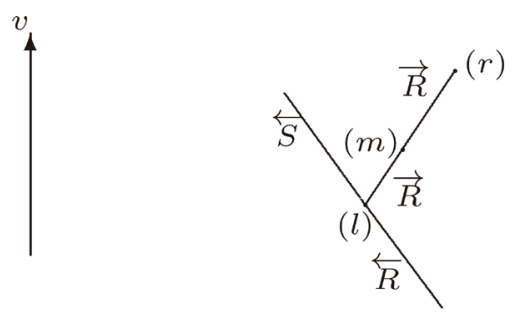

Figure 13. Overtaking of $\vec{R}$ and $\vec{R}$.

\section{Case 3: The collision of $\vec{W}$ and J.}

For this case, we have

$$
\left\{\begin{array}{l}
\vec{W} \text { or } \bar{W}: \frac{\mathrm{d} x}{\mathrm{~d} t}=u_{m} \pm \frac{s}{\rho_{m}} \\
J: \frac{\mathrm{d} x}{\mathrm{~d} t}=u_{m}=u_{r}
\end{array}\right.
$$


It follows that the backward wave $\bar{W}$ cannot intersect with the contact discontinuity $J$ in a finite time. We just need to consider the intersection of the forward wave $\vec{W}$ and the contact discontinuity $J$ which will interect with each other ar the finite time $t=t_{0}$ (Figure 14).

Subcase $3.1 \vec{R}$ and $J, v_{*}>0$ (Figure 15).

For this subcase, we have

$$
\left\{\begin{array}{l}
\vec{W}\left(Q_{l} Q_{m}\right): u_{m}=u_{l}+s\left(v_{m}-v_{l}\right), \\
J: u_{m}=u_{r}, v_{m}=v_{r}, \rho_{m} \neq \rho_{r}, \\
\overleftarrow{W}\left(Q_{l} Q_{*}\right): u_{*}=u_{l}-s\left(v_{*}-v_{l}\right), \\
\vec{W}\left(Q_{*} Q_{r}\right): u_{*}=u_{r}+s\left(v_{*}-v_{r}\right),
\end{array}\right.
$$

then $\vec{R}$ will intersect with $J$ at the finite time $t=t_{0}$ and a new Riemann problem is formed.

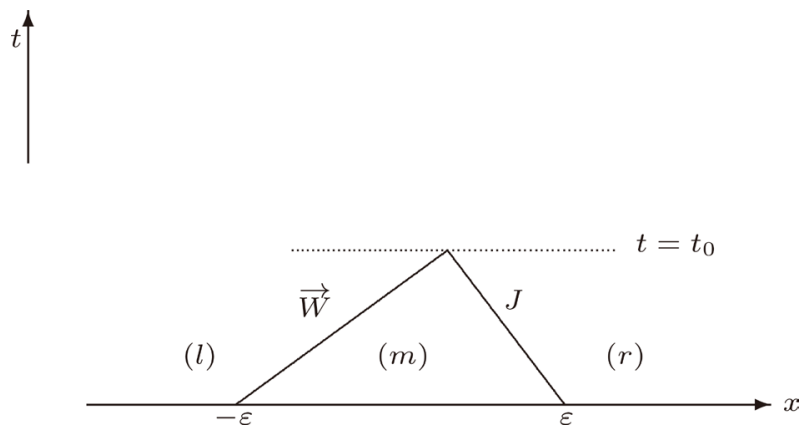

Figure 14. Interaction of $\vec{W}$ and $J$.
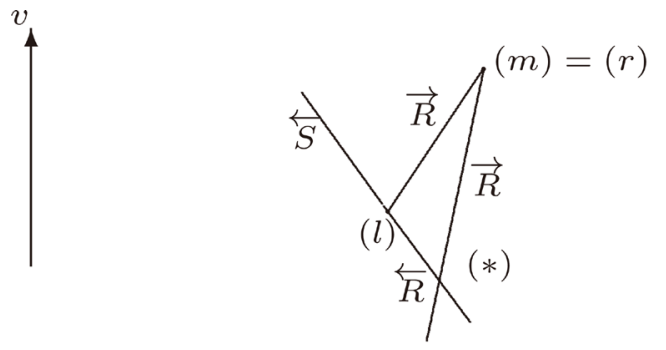

Figure 15. Intersection of $\vec{R}$ and $J, v_{*}>0$.

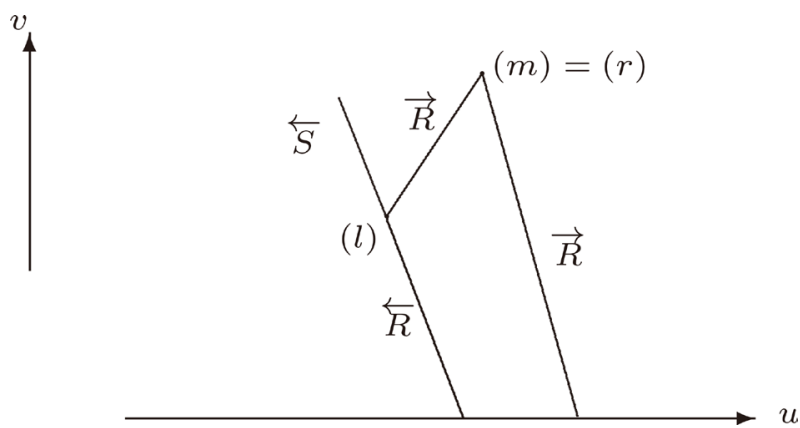

Figure 16. Intersection of $\vec{R}$ and $J, v_{*} \leq 0$. 


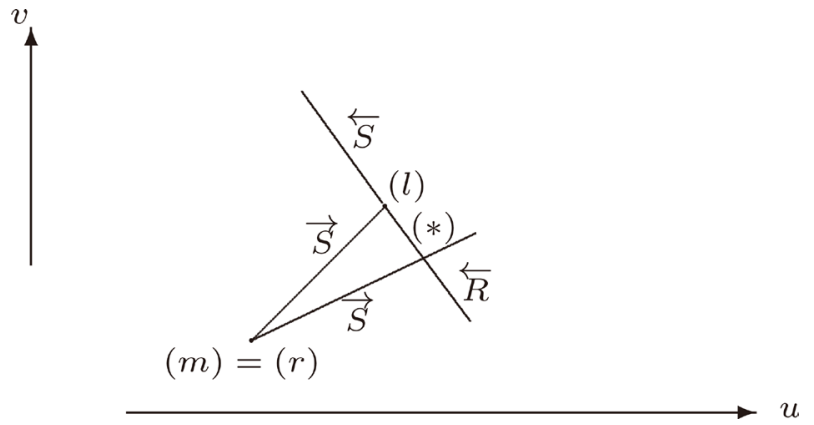

Figure 17. Intersection of $\vec{S}$ and $J$.

From the left state we draw the backward wave curve $\bar{W}\left(Q_{l}\right)$, and from the right state we draw the forward wave curve $\vec{W}\left(Q_{r}\right)$, they intersect with each other at $Q_{*} . Q_{*}\left(u_{*}, v_{*}\right)$ is the projection of the state $\left(\rho_{* 1}, u_{*}, v_{*}\right)$ and the state $\left(\rho_{* 2}, u_{*}, v_{*}\right)$ on the plane $(u, v)$, where $\rho_{* 1}=\frac{s}{u_{*}-u_{m}+\frac{s}{\rho_{m}}}$ and $\rho_{* 2}=\frac{s}{u_{*}-u_{m}+\frac{s}{\rho_{r}}}$. Since $\rho_{m} \neq \rho_{r}$, it follows that it is the contact discontinuity $J$ which connects the state $\left(\rho_{* 1}, u_{*}, v_{*}\right)$ with the state $\left(\rho_{* 2}, u_{*}, v_{*}\right)$.

Thus for this subcase, the unique solution of the new Riemann problem is given by

$$
\vec{R}+J \rightarrow \bar{R} \text { or } \bar{S}+J+\vec{R} .
$$

Subcase $3.2 \vec{R}$ and $J, v_{*} \leq 0$ (Figure 16).

For this subcase, since $v_{*} \leq 0$, we know that the positional relations of the left state and the right state are shown in Figure 16. From the left state we draw the backward wave curve $\bar{W}\left(Q_{l}\right)$, and from the right state we draw the forward wave curve $\vec{W}\left(Q_{r}\right)$, we have to consider the delta shock wave solution. Then the unique solution is given as follows $\vec{R}+J \rightarrow \delta$.

Subcase $3.3 \vec{S}$ and $J$ (Figure 17).

Similar discussions as in Subcase 3.1, we obtain the unique solution of the wave interaction for this subcase is given by

$$
\vec{S}+J \rightarrow \bar{S} \text { or } \bar{R}+J+\vec{S} \text {. }
$$

Theorem 3.3. When the forward shock wave collides with the contact discontinuity $J$, the forward shock wave will pass through the contact discontinuity, and the backward shock (or rarefaction) wave will occur propagating in the opposite direction. When the forward rarefaction wave collides with the contact discontinuity $J$, there are two possibilities. One is that the forward rarefaction wave will pass through the contact discontinuity, and the backward shock (or rarefaction) wave will occur propagating in the opposite direction, the other one is that the solution of the new Riemann problem is the delta shock wave. The above conclusions reveal that the Riemann solution of (1) with the initial data 
(4) are stable for this case.

Now we construct the unique solution of the elementary wave interactions and get the following main conclusion.

Theorem 3.4. The unique solution of the initial value problem (1) with the initial values (3) is constructed case by case. By analyzing the wave structures, we conclude that the Riemann solutions of the system (1) with the initial data (4) are stable under such small perturbation on the initial data (4).

\section{Conclusion}

Using the characteristic analysis method, i.e., by analyzing the elementary wave curves in the phrase plane, we get the unique solution of the initial problem (1) and the initial values (3). We observe that the elementary wave interactions have a much simpler structure for Temple class than general systems of conservation laws since the wave interaction of the same family does not generate a wave of other family for Temple systems. We observe that when the delta shock wave does not occur at the time $t=0$, the delta shock wave may occur at the finite time for some cases of the initial value problem (1) and (3).

\section{Conflicts of Interest}

The authors declare no conflicts of interest regarding the publication of this paper.

\section{References}

[1] Bouchut, F. (2004) Nonlinear Stability of Finite Volume Methods for Hyperbolic Conservation Laws and Well-Balanced Schemes for Sources. Frontiers in Mathematics, Birkhäuser, Basel, Switzerland.

[2] Bouchut, F. and Boyaval, S. (2013) A New Model for Shallow Viscoelastic Fluids. Mathematical Models and Methods in Applied Sciences, 23, 1479-1526. https://doi.org/10.1142/S0218202513500140

[3] Carbou, G., Hanouzet, B. and Natalini, R. (2009) Semiliear Behavior for Totally Linearly Degenerate Hyperbolic Systems with Relaxation. Journal of Differential Equations, 246, 291-319. https://doi.org/10.1016/j.jde.2008.05.015

[4] Suliciu, I. (1990) On Modelling Phase Transitions by Means of Ratetype Constitutive Equations, Shock Wave Structure. International Journal of Engineering Science, 28, 829-841. https://doi.org/10.1016/0020-7225(90)90028-H

[5] Chen, T.T., Qu, A.F. and Wang, Z. (2017) The Two-Dimensional Riemann Problem for Isentropic Chaplygin Gas. Acta Mathematica Scientia, 37A, 1053-1061.

[6] Wang, Q., Zhang, J.Q. and Yang, H.C. (2020) Two Dimensional Riemann-Type Problem and Shock Diffraction for the Chaplygin Gas. Applied Mathematics Letters, 101, Article 106046. https://doi.org/10.1016/j.aml.2019.106046

[7] Temple, B. (1983) Systems of Conservation Laws with Coinciding Shock and Rarefaction Curves. Contemporary Mathematics, 17, 143-151.

https://doi.org/10.1090/conm/017/16

[8] Chalons, C. and Coquel, F. (2005) Navier-Stokes Equations with Several Independent Pressure Laws and Explicit Predictor-Corrector Schemes. Numerische Mathe- 
matik, 101, 451-478. https://doi.org/10.1007/s00211-005-0612-7

[9] De la cruz, R., Galvis, J., Juajibioy, J.C. and Rendón, L. (2014) Delta Shock Wave for the Suliciu Relaxation System. Advances in Mathematical Physics, 2014, Article ID 354349. https://doi.org/10.1155/2014/354349

[10] Chang, T. and Hsiao, L. (1989) The Riemann Problem and Interaction of Waves in Gas Dynamics, Pitman Monographs, No. 41, Longman Scientific and Technical, Essex.

[11] Zhu, L. (2010) Chaplygin Gas Dynamic System in Adiabatic Flow. Master Thesis, Shanghai University, Shanghai. 